\title{
ITERATING THE BASIC CONSTRUCTION
}

\author{
MIHAI PIMSNER AND SORIN POPA
}

\begin{abstract}
Let $N \subset M$ be a pair of type $\mathrm{II}_{1}$ factors with finite Jones' index and $N \subset M \subset M_{1} \subset M_{2} \subset \cdots \subset M_{n} \subset \cdots \subset M_{2 n+1}$ be the associated tower of type $\mathrm{II}_{1}$ factors obtained by iterating Jones' basic construction. We give an explicit formula of a projection in $M_{2 n+1}$ which implements the conditional expectation of $M_{n}$ onto $N$, thus showing that $M_{2 n+1}$ comes naturally from the basic construction associated to the pair $N \subset M_{n}$. From this we deduce several properties of the relative commutant $N^{\prime} \cap M_{n}$.
\end{abstract}

Introduction. Let $N \subset M$ be a pair of finite factors. Jones defined in [1] the index $[M: N]$ of $N$ in $M$ to be the coupling constant of $N$ in its representation on $L^{2}(M)$. If this index is finite, then the trace preserving conditional expectation of $M$ onto $N$, regarded as an operator on $L^{2}(M)$, generates together with $M$ a finite factor $M_{1}$. This factor is called in Jones' terminology the extension of $M$ by $N$ and the construction of $M_{1}$ from $M$ and $N$, the basic construction. The pair $M \subset M_{1}$ has the remarkable property that $\left[M_{1}: M\right]=[M: N]$, so this procedure may be iterated to get an increasing sequence of finite factors $N \subset M \subset M_{1} \subset M_{2} \subset \cdots$ and together with it a sequence of projections $e_{i} \in M_{i+1}, i \geq 0$, implementing the conditional expectations at consecutive steps.

We prove in this paper that in this sequence of factors the basic construction arises periodically from $n$ to $n$ steps, for any $n$. In fact we give a formula for a projection $f_{n}$ in $M_{2 n+1}$ that implements the conditional expectation of $M_{n}$ onto $N: f_{n}$ is a scalar multiple of the word of maximal length in $\left\{e_{i}\right\}_{0 \leq i \leq 2 n}$, namely

$$
f_{n}=[M: N]^{n(n+1) / 2}\left(e_{n} e_{n-1} \cdots e_{0}\right)\left(e_{n+1} e_{n} \cdots e_{1}\right) \cdots\left(e_{2 n} \cdots e_{n}\right) .
$$

We mention that this result was independently obtained by A. Ocneanu [2]. We apply this theorem to show that if the logarithm of the index $[M: N]$ equals the relative entropy $H(M \mid N)$ considered in [3], then one also has

$$
H\left(M_{n} \mid N\right)=\ln \left[M_{n}: N\right] \text { for every } n .
$$

Since this equality characterizes an extremal case for an inclusion of factors, from the analysis of a similar situation in [3] we deduce several properties of the inclusion $N \subset M_{n}$ and of the relative commutant $N^{\prime} \cap M_{n}$.

1. Preliminaries. Throughout this paper $M$ will be a finite factor with normalized trace $\tau, \tau(1)=1$. We denote by $\|x\|_{2}=\tau\left(x^{*} x\right)^{1 / 2}, x \in M$, the Hilbert norm given by $\tau$ and by $L^{2}(M, \tau)$ the Hilbert space completion of $M$ in this norm. The canonical conjugation of $L^{2}(M, \tau)$ is denoted by $J$. It acts on $M \subset L^{2}(M, \tau)$

Received by the editors February 23, 1987.

1980 Mathematics Subject Classification (1985 Revision). Primary 46L35; Secondary 46L10. 
by $J x=x^{*}$ and satisfies $J M J=M^{\prime}$. In fact, if we regard $M$ as acting by left multiplication on $L^{2}(M, \tau)$ then for $x \in M, J x J$ is the operator of right multiplication by $x^{*}$.

$N \subset M$ will denote a subfactor of $M$ with $1_{N}=1_{M}$ and $E_{N}$ will be the unique normal trace preserving conditional expectation of $M$ onto $N$. Note that $E_{N}$ is just the restriction to $M \subset L^{2}(M, \tau)$ of the orthogonal projection $e_{N}$ of $L^{2}(M, \tau)$ onto $L^{2}(N, \tau)$ (the closure of $N$ in $L^{2}(M, \tau)$ ). The conditional expectation $E_{N}$, the projection $e_{N}$ and the conjugation $J$ are related by the properties

(i) If $x \in M$ then $x \in N$ iff $e_{N} x=x e_{N}$.

(ii) $e_{N} x e_{N}=E_{N}(x) e_{N}, x \in M$.

(iii) $J$ commutes with $e_{N}$.

If the index of $N$ in $M$ is finite then from the pair $N \subset M$ one can construct a new pair of finite factors $M \subset M_{1}$ with the same index $\left[M_{1}: M\right]=[M: N]$. The construction of $M_{1}$ is called the basic construction and the factor $M_{1}$ is called the extension of $M$ by $N$.

We recall from [1] the definition and main properties of $M_{1}$ :

1.1 Proposition. Define $M_{1}=J N^{\prime} J$. Then we have

$1^{\circ} M_{1}=\left(M \cup\left\{e_{N}\right\}\right)^{\prime \prime}$,

$2^{\circ}\left[M_{1}: M\right]=[M: N]$ and if $\tau$ denotes the unique normalized trace on $M_{1}$ and $E_{M}$ the $\tau$ preserving conditional expectation of $M_{1}$ onto $M$, then $E_{M}\left(e_{N}\right)=$ $[M: N]^{-1} 1_{M}$ or equivalently $\tau\left(e_{N} x\right)=[M: N]^{-1} \tau(x)$ for every $x \in M$.

Part $1^{\circ}$ of this proposition can be made more precise: by [3], if $n+1 \geq[M: N]$ then any element in $M_{1}$ is a sum of at most $(n+1)^{2}$ monomials of the form $x e_{N} y, x, y \in M$. Note that $M_{1}$ can also be described abstractly as the unique (up to isomorphism) finite factor $M_{1}$ which contains $M$ and a projection $e$ so that $\left[M_{1}: M\right]=[M: N],[e, y]=0$ for $y \in N$, exe $=E_{N}(x) e$ for $x \in M$, and with the trace $\tau$ satisfying $\tau(e x)=\left[M_{1}: M\right]^{-1} \tau(x), x \in M$. In fact one of the conditions is redundant: the next proposition gives two equivalent ways of characterizing $M_{1}$.

1.2 Proposition. Let $N \subset M$ be a pair of finite factors with finite index and $M_{1}$ the extension of $M$ by $N$. Let $\tilde{M}$ be a finite factor that contains $M$ and with normalized trace $\tilde{\tau}, E_{M}$ the $\tilde{\tau}$-preserving conditional expectation of $\tilde{M}$ onto $M$ and $e \in \tilde{M}$ an orthogonal projection. Then the following conditions are equivalent:

$1^{\circ}$ There exists an isomorphism $\phi$ of $M_{1}$ onto $\tilde{M}$ such that $\phi(x)=x$ for $x \in M$ and $\phi\left(e_{N}\right)=e$.

$2^{\circ}$ (i) $[e, y]=0, y \in N$;

(ii) $E_{M}(e)=[\tilde{M}: M]^{-1} 1_{M}=[M: N]^{-1} 1_{M}$.

$3^{\circ}$ (i) $e x e=E_{N}(x) e, x \in M$, and $e \neq 0$;

(ii) $e$ and $M$ generate $\tilde{M}$ as a von Neumann algebra.

PROOF. $1^{\circ}$ implies $2^{\circ}$ by the known properties of $e_{N}$.

Suppose $2^{\circ}$ holds. Then by 1.8 of $[\mathbf{3}]$ we get that $\tilde{M}$ is the extension of $M$ by $P$ where $P=\{e\}^{\prime} \cap M$. But (i) implies that $N \subset P$ and since $[M: P]=[\tilde{M}: M]=$ $[M: N]$ we conclude that $N=P$. Thus $e$ and $M$ generate $\tilde{M}$ as a von Neumann algebra and again by 1.8 of [3] we get $E_{N}(x) e=e x e$, for every $x \in M$. 
Assume that $3^{\circ}$ holds. Using the "orthonormal basis" of [3] it is easy to see that the map $\phi: M_{1} \rightarrow \tilde{M}$ that sends $\sum x_{i} e_{N} y_{i}$ to $\sum x_{i} e y_{i}$ is a well-defined *homomorphism. Moreover $\phi$ satisfies $m \phi(x)=\phi(m x)$ for every $m \in M$ and $x \in M_{1}$. This shows that $\phi(1)$ is a projection that commutes with $e$ and with every $m \in M$. By (ii) we conclude that $\phi(1)$ is central and since $e \neq 0$ and $M$ is a factor $\phi(1)=1$. This implies now that $\phi(m)=\phi(m 1)=m \phi(1)=m$ and since obviously $\phi\left(e_{N}\right)=e$ we get $1^{\circ}$. Q.E.D.

The pair $M \subset M_{1}$ having finite index one can construct its extension $M_{1} \subset M_{2}$ and in fact the whole procedure may be iterated to get an increasing sequence of finite factors $N \subset M \subset M_{1} \subset M_{2} \subset \cdots$, and orthogonal projections $e_{i} \in M_{i+1}$, $i \geq 0\left(N=M_{-1}, M=M_{0}\right)$ in which $M_{i+1}$ is the extension of $M_{i}$ by $M_{i-1}$ or in other words $M_{i+1}$ and $e_{i}$ are obtained by the basic construction from the pair $M_{i-1} \subset M_{i}$. Thus if $\tau$ denotes the unique normalized trace on $\bigcup_{i} M_{i}$ and $E_{M_{i-1}}$ the $\tau$-preserving conditional expectation of $M_{i}$ onto $M_{i-1}, i \geq 0$, then:

(a) $\left[e_{i}, y\right]=0$ for $y \in M_{i-1}$;

(b) $e_{i} x e_{i}=E_{M_{i-1}}(x) e_{i}, x \in M_{i}$;

(c) $\left[M_{i+1}: M_{i}\right]=[M: N]$ and $E_{M_{i}}\left(e_{i}\right)=[M: N]^{-1} 1$.

In particular it follows that the sequence of projections $e_{i}$ satisfies $\left[e_{i}, e_{j}\right]=0$, $|i-j| \geq 2, e_{i} e_{i \pm 1} e_{i}=[M: N]^{-1} e_{i}$ and $\tau\left(e_{i} w\right)=[M: N]^{-1} \tau(w)$ for every word in $1, e_{0}, e_{1}, \ldots, e_{i-1}$.

2. $n$-step extensions. In this section we prove the main result of the paper: we show that if $N \subset M \subset M_{1} \subset \cdots$ is the sequence of finite factors obtained by iterating the basic construction as in $\S 1$, then, for each $n>0, M_{2 n+1}$ is the extension of $M_{n}$ by $N$. In fact we give an explicit formula for a projection $f_{n} \in$ $M_{2 n+1}$ which implements the conditional expectation of $M_{n}$ onto $N$ and generates with $M_{n}$ the factor $M_{2 n+1}: f_{n}$ will be a scalar multiple of the word of maximal length in $e_{0}, e_{1}, \ldots, e_{2 n}$ where $e_{i} \in M_{i+1}$ are as in $\S 1$.

We define for each $n, k \geq 0$ the element

$$
g_{n}^{k}=\left(e_{n+k} e_{n+k-1} \cdots e_{k}\right)\left(e_{n+k+1} e_{n+k} \cdots e_{k+1}\right) \cdots\left(e_{2 n+k} e_{2 n+k-1} \cdots e_{n+k}\right)
$$

(there are $n+1$ products of parentheses and in each parentheses the product of $n+1$ consecutive projections $e_{i}$ in decreasing order). We put $f_{n}^{k}=[M: N]^{n(n+1) / 2} g_{n}^{k} \in$ $M_{2 n+k+1}$ and $f_{n}=f_{n}^{0} \in M_{2 n+1}$.

To prove that the above defined $f_{n}$ implements the basic construction in the extension of $M_{n}$ by $N$, we only have to show that $f_{n}$ is an orthogonal projection, that $f_{n} \in N^{\prime} \cap M_{2 n+1}$ and that $E_{M_{n}}\left(f_{n}\right)=\left[M_{n}: N\right]^{-1}=\left[M_{2 n+1}: M_{n}\right]^{-1}$. (See Proposition 1.2.) Note that since $\left[M_{i+1}: M_{i}\right]=[M: N]$, by the multiplicative property of the index we do have $\left[M_{n}: N\right]=[M: N]^{n+1}=\left[M_{2 n+1}: M_{n}\right]$. To prove the other properties, let us first recall some facts about the algebra generated by $\left\{e_{i}\right\}_{i \geq 0}$ (cf. [1]).

A finite product of $e_{i}$ 's is called a word. It is called a reduced word if it is of minimal length for the grammatical rules $e_{i} e_{i \pm 1} e_{i} \leftrightarrow e_{i}, e_{i}^{2} \leftrightarrow e_{i}$ and $e_{i} e_{j} \leftrightarrow e_{j} e_{i}$ for $|i-j| \geq 2$. Note that any word is a scalar multiple of a reduced word. Jones pointed out (in $[\mathbf{1}, 4.1 .4])$ that reduced words can be uniquely written in the ordered form

$$
w=\left(e_{j_{1}} e_{j_{1}-1} \cdots e_{k_{1}}\right)\left(e_{j_{2}} e_{j_{2}-1} \cdots e_{k_{2}}\right) \cdots\left(e_{j_{p}} e_{j_{p}-1} \cdots e_{k_{p}}\right)
$$

where $j_{i} \geq k_{i}, j_{i+1}>j_{i}, k_{i+1}>k_{i}$. 
From this description of reduced words it follows that if a reduced word $w$ is written with the letters $e_{r}, e_{r+1}, \ldots, e_{s}(s \geq r)$ then $e_{r+i}$ and $e_{s-i}$ appear at most $i+1$ times in $w$.

To prove the theorem we first show that $g_{n}^{0}$ are selfadjoint elements. This will be an easy consequence of the next two lemmas.

2.1 LEMMA. $g_{n}^{0}$ is the unique reduced word of maximal length in $e_{0}, e_{1}, \ldots, e_{2 n}$.

PROOF. Since by definition $g_{n}^{0}$ is of the form $(*)$ it is a reduced word. As noted before if $w$ is an arbitrary reduced word in $e_{0}, e_{1}, \ldots, e_{2 n}$ then $e_{0}, e_{2 n}$ appear at most once in $w, e_{1}, e_{2 n-1}$ at most twice and more generally $e_{k}, e_{2 n-k}$ at most $k+1$ times. Thus the length of $w$ is at most equal to $1+2+\cdots+n+(n+1)+n+\cdots+2+1$ and by inspecting the conditions $j_{i} \geq k_{i}, j_{i+1}>j_{i}, k_{i+1}>k_{i}$ of $(*)$ it follows that the only reduced word $w$ with this length is obtained when $j_{i}=n+i, k_{i}=i$, i.e. $w=g_{n}^{0}$. Q.E.D.

2.2 LEMMA. If $w$ is a reduced word in $e_{0}, e_{1}, \ldots, e_{2 n}$ then the reduced form of $w^{*}$ has the same length as $w$.

ProOF. Indeed, $w^{*}$ has length at most equal to that of $w$ and since $\left(w^{*}\right)^{*}=w$, the statement follows. Q.E.D.

To prove that $g_{n}^{0}$ are scalar multiples of projections we have to compute $\left(g_{n}^{0}\right)^{2}$. To do this we use an induction argument based on the formula

2.3 LEMMA. $g_{n}^{0}=\left(e_{n} e_{n+1} \cdots e_{2 n}\right) g_{n-1}^{0}\left(e_{2 n-1} \cdots e_{n}\right)$.

ProOF. The equality follows by pushing $e_{2 n}$ to the left as much as possible in the formula giving $g_{n}^{0}$. Q.E.D.

2.4 REMARK. Two other equalities that can be obtained in a similar fashion and seem to be of interest are

$$
g_{n}^{0}=g_{n-1}^{1}\left(e_{2 n} \cdots e_{n+1}\right)\left(e_{0} \cdots e_{n}\right)=\left(e_{n} e_{n-1} \cdots e_{0}\right) g_{n-1}^{2}\left(e_{1} e_{2} \cdots e_{n}\right) .
$$

To show that $g_{n}^{0}$ projects on a scalar in $M_{n}$ we prove

2.5 LEMMA. $E_{M_{2 n}}\left(g_{n}^{0}\right)=[M: N]^{-(n+1)} g_{n-1}^{1}$. More generally

$$
E_{M_{2 n+k}}\left(g_{n}^{k}\right)=[M: N]^{-(n+1)} g_{n-1}^{k+1} \text {. }
$$

PROOF. It is enough to prove that $E_{M_{2 n}}\left(g_{n}^{0}\right)=\lambda^{n+1} g_{n-1}^{1}$, where $\lambda=[M: N]^{-1}$, because the rest of the statement follows by starting the sequence of factors from $M_{k-1} \subset M_{k}$, instead of $N=M_{-1} \subset M_{0}=M$.

We first show that for $j \geq p \geq k+1$ we have

$$
\left(e_{j} e_{j-1} \cdots e_{k}\right)\left(e_{p} e_{p-1} \cdots e_{k+1}\right)=\lambda\left(e_{p-2} \cdots e_{k}\right)\left(e_{j} \cdots e_{k+1}\right) .
$$

Indeed we have

$$
\begin{aligned}
\left(e_{j} e_{j-1} \cdots e_{p} e_{p-1} \cdots e_{k}\right) e_{p} & =\lambda\left(e_{j} e_{j-1} \cdots e_{p}\right)\left(e_{p-2} e_{p-3} \cdots e_{k}\right) \\
& =\lambda\left(e_{p-2} \cdots e_{k}\right)\left(e_{j} e_{j-1} \cdots e_{p}\right)
\end{aligned}
$$


which easily implies (**). Applying recursively (**) we get

$$
\begin{aligned}
E_{M_{2 n}}\left(g_{n}^{0}\right) & =\left(e_{n} e_{n-1} \cdots e_{0}\right) \cdots\left(e_{2 n-1} \cdots e_{n-1}\right) E_{M_{2 n}}\left(e_{2 n}\right)\left(e_{2 n-1} \cdots e_{n}\right) \\
& =\lambda\left(e_{n} \cdots e_{0}\right) \cdots\left(e_{2 n-1} \cdots e_{n-1}\right)\left(e_{2 n-1} \cdots e_{n}\right) \\
& =\lambda^{2}\left(e_{n} \cdots e_{0}\right) \cdots\left(e_{2 n-2} e_{2 n-3} \cdots e_{n-2}\right)\left(e_{2 n-3} \cdots e_{n-1}\right)\left(e_{2 n-1} \cdots e_{n}\right) \\
& =\lambda^{3}\left(e_{n} \cdots e_{0}\right) \cdots\left(e_{2 n-5} \cdots e_{n-2}\right)\left(e_{2 n-2} \cdots e_{n-1}\right)\left(e_{2 n-1} \cdots e_{n}\right) \\
& =\cdots=\lambda^{n}\left(e_{n} \cdots e_{0}\right) e_{1}\left(e_{n+1} \cdots e_{2}\right) \cdots\left(e_{2 n-2} \cdots e_{n-1}\right)\left(e_{2 n-1} \cdots e_{n}\right) \\
& =\lambda^{n+1}\left(e_{n} \cdots e_{1}\right)\left(e_{n+1} \cdots e_{2}\right) \cdots\left(e_{2 n-1} \cdots e_{n}\right)=\lambda^{n+1} g_{n-1}^{1} \cdot \text { Q.E.D. }
\end{aligned}
$$

We can now prove the theorem.

2.6 THEOREM. Let $N \subset M$ be a pair of finite factors with $[M: N]<\infty$. Let $N \subset M \subset M_{1} \subset \cdots$ be the sequence of finite factors obtained by iterating the basic construction and $e_{i} \in M_{i+1}$ the projection implementing the conditional expectation of $M_{i}$ onto $M_{i-1}$ at each step of the basic construction as in $\S 1$, for $i \geq 0\left(M_{-1}=N, M_{0}=M\right)$. Let

$$
f_{n}=[M: N]^{n(n+1) / 2}\left(e_{n} e_{n-1} \cdots e_{0}\right)\left(e_{n+1} e_{n} \cdots e_{1}\right) \cdots\left(e_{2 n} e_{2 n-1} \cdots e_{n}\right) \in M_{2 n+1} \text {. }
$$

Then $M_{2 n+1}$ is the extension of $M_{n}$ by $N$ and $f_{n} \in M_{2 n+1}$ is the projection that implements the conditional expectation of $M_{n}$ onto $N$, i.e. $f_{n} \in N^{\prime} \cap M_{2 n+1}$, $f_{n} x f_{n}=E_{N}(x) f_{n}, x \in M_{n}, E_{M_{n}}\left(f_{n}\right)=\left[M_{n}: N\right]^{-1}$ and $M_{2 n+1}=\left(M_{n} \cup\left\{f_{n}\right\}\right)^{\prime \prime}$.

PROOF. We will prove the theorem by induction over $n \geq 0$. If $n=0$ then $f_{0}=e_{0}$ and we have nothing to prove. Assume the statement is true up to $n-1$. Let $\lambda=[M: N]^{-1}$ and $c_{n}=\lambda^{-n(n+1) / 2}$. Since $f_{n}=c_{n} g_{n}^{0}$ and $g_{n}^{0}$ is a word in $e_{0}, e_{1}, \ldots, e_{2 n}$, which all commute with $N$, it follows that $f_{n} \in N^{\prime} \cap M_{2 n+1}$. Note also that since $e_{2 n} \in M_{2 n-1}^{\prime} \cap M_{2 n+1}, e_{2 n}$ commutes with $g_{n-1}^{0} \in M_{2 n-1}$. To see that $g_{n}^{0}$ is selfadjoint we use Lemma 2.2 to obtain that $g_{n}^{0 *}$ has the same length as $g_{n}^{0}$ and thus by Lemma $2.1 g_{n}^{0}=\left(g_{n}^{0}\right)^{*}$. Further, Lemma 2.3 implies that

$$
\begin{aligned}
\left(g_{n}^{0}\right)^{2} & =g_{n}^{0 *} g_{n}^{0} \\
& =\left(e_{n} e_{n+1} \cdots e_{2 n-1}\right) g_{n-1}^{0}\left(e_{2 n} e_{2 n-1} \cdots e_{n+1} e_{n} e_{n+1}\right. \\
& \left.\cdots e_{2 n-1} e_{2 n}\right) g_{n-1}^{0}\left(e_{2 n-1} \cdots e_{n}\right) \\
& =\lambda^{n}\left(e_{n} e_{n+1} \cdots e_{2 n-1}\right) g_{n-1}^{0} e_{2 n} g_{n-1}^{0}\left(e_{2 n-1} \cdots e_{n}\right) \\
& =\lambda^{n}\left(e_{n} e_{n+1} \cdots e_{2 n}\right)\left(g_{n-1}^{0}\right)^{2}\left(e_{2 n-1} \cdots e_{n}\right) \\
& =\lambda^{n} c_{n-1}^{-1}\left(e_{n} e_{n+1} \cdots e_{2 n}\right) g_{n-1}^{0}\left(e_{2 n-1} \cdots e_{n}\right) \\
& =\lambda^{n} c_{n-1}^{-1} g_{n}^{0}=c_{n}^{-1} g_{n}^{0} .
\end{aligned}
$$

Thus $f_{n}=c_{n} g_{n}^{0}$ is a selfadjoint projection in $N^{\prime} \cap M_{2 n+1}$. Next we apply recursively Lemma 2.5 to get

$$
\begin{aligned}
E_{M_{n}}\left(f_{n}\right) & =c_{n} E_{M_{n}}\left(g_{n}^{0}\right)=c_{n} E_{M_{n}} E_{M_{2 n}}\left(g_{n}^{0}\right)=c_{n} \lambda^{n+1} E_{M_{n}}\left(g_{n-1}^{1}\right) \\
& =c_{n} \lambda^{n+1} E_{M_{n}} E_{M_{2 n-1}}\left(g_{n-1}^{1}\right)=c_{n} \lambda^{(n+1)+n} E_{M_{n}}\left(g_{n-2}^{2}\right) \\
& =\cdots=c_{n} \lambda^{(n+1)+n+\cdots+2} E_{M_{n}}\left(g_{0}^{n}\right)=c_{n} \lambda^{(n+1)+n+\cdots+2} E_{M_{n}}\left(e_{n}\right) \\
& =c_{n} \lambda^{(n+2)(n+1) / 2} 1_{M_{n}}=\lambda^{n+1} 1_{M_{n}}
\end{aligned}
$$

(we used $g_{0}^{n}=e_{n}$ ). 
Moreover by [1],

$$
\begin{aligned}
{\left[M_{2 n+1}: M_{n}\right] } & =\prod_{n \leq i \leq 2 n}\left[M_{i+1}: M_{i}\right]=[M: N]^{n+1} \\
& =\prod_{0 \leq i \leq n}\left[M_{i+1}: M_{i}\right]=\left[M_{n}: N\right] .
\end{aligned}
$$

By Proposition 1.2 the rest of the properties of $f_{n}$ follow automatically. Q.E.D.

2.7 REMARK. We could include the proof of $g_{n}^{0}=g_{n}^{0 *}$ in the induction argument. Indeed by Lemma 2.3 and using $g_{n-1}^{0}=\left(g_{n-1}^{0}\right)^{*}$ and $\left[e_{2 n}, g_{n-1}^{0}\right]=0$ we get

$$
\begin{aligned}
\left(g_{n}^{0}\right)^{*} & =e_{n} e_{n+1} \cdots e_{2 n}\left(g_{n-1}^{0}\right)^{*} e_{2 n-1} e_{2 n-2} \cdots e_{n} \\
& =e_{n} e_{n+1} \cdots e_{2 n} g_{n-1}^{0} e_{2 n-1} \cdots e_{n}=g_{n}^{0} .
\end{aligned}
$$

We preferred however the deductive argument of Lemmas 2.1 and 2.2 as it points out some properties of $f_{n}$.

3. Some applications. In this section we derive some consequences on the inclusion $N \subset M_{n}$. We consider the case when the relative entropy $H(M \mid N)$ considered in [3] satisfies $H(M \mid N)=\ln [M: N]$. An important case when this equality occurs is when $N^{\prime} \cap M=\mathrm{C}$ (cf. [3]). First we compute the relative entropy from $n$ to $n$ steps.

3.1 THEOREM. If $H(M \mid N)=\ln [M: N]$ then

$$
H\left(M_{n+k} \mid M_{k-1}\right)=\ln \left[M_{n+k}: M_{k-1}\right], \quad \text { for every } n, k \geq 0 .
$$

In particular $H\left(M_{n} \mid N\right)=\ln \left[M_{n}: N\right]$ and $H\left(M_{k} \mid M_{k-1}\right)=\ln \left[M_{k}: M_{k-1}\right]$, for every $k \geq 0$.

ProOF. Since $H(M, N)=\ln [M: N], E_{N^{\prime} \cap M}\left(e_{0}\right)=\lambda 1$ and the anti-isomorphism $N^{\prime} \cap M \ni x^{\prime} \mapsto \theta_{0}\left(x^{\prime}\right)=J_{M} x^{\prime} J_{M} \in M^{\prime} \cap M_{1}$ is trace preserving (cf. 4.5 in [3]). To show that $E_{M^{\prime} \cap M_{1}}\left(e_{1}\right)=\lambda 1$ it suffices to prove that $M^{\prime} \cap M_{1} \ni y^{\prime} \mapsto$ $\theta_{1}\left(y^{\prime}\right)=J_{M_{1}} y^{\prime} J_{M_{1}} \in M^{\prime} \cap M_{1}$ is also trace preserving (cf. [3]). But $\theta_{1} \theta_{0}=\sigma^{\prime}$, where $\sigma^{\prime}$ is the restriction to $N^{\prime} \cap M$ of the isomorphism $\sigma$ defined in [3, 1.3], $\sigma^{\prime}\left(x^{\prime}\right)=\lambda^{-1} \sum_{i} m_{i} e_{0} e_{1} x^{\prime} e_{0} m_{i}^{*}$, with $\left\{m_{i}\right\}$ an orthonormal basis of $M$ over $N$. Indeed if $x^{\prime}=\sum_{i} m_{i} n_{i} \in N^{\prime} \cap M$, with $n_{i} \in N$, then $\theta_{0}\left(x^{\prime}\right) \in M_{1}$ implies $\theta_{0}\left(x^{\prime}\right)=$ $\sum_{i, j} m_{i} E_{N}\left(m_{i}^{*} m_{j} x^{\prime *}\right) e_{N} m_{j}^{*}$ and thus in $L^{2}\left(M_{1}, \tau\right)$ we have

$$
\begin{aligned}
\theta_{1}\left(\theta_{0}\left(x^{\prime}\right)\right)\left(m_{p} n e_{0} m_{r}^{*}\right) & =\sum_{i, j} m_{p} n e_{0} m_{r}^{*} m_{j} E_{N}\left(x^{\prime} m_{j}^{*} m_{i}\right) e_{0} m_{i}^{*} \\
& =\sum_{i} m_{p} n e_{0} E_{N}\left(x^{\prime} m_{r}^{*} m_{i}\right) m_{i}^{*}=m_{p} n e_{0} x^{\prime} m_{r}^{*} \\
& =m_{p} e_{0} x^{\prime} n m_{r}^{*}=\sigma^{\prime}\left(x^{\prime}\right)\left(m_{p} n e_{0} m_{r}^{*}\right),
\end{aligned}
$$

for all $n \in N$. Thus, since $\sigma^{\prime}, \theta_{0}$ are trace preserving, $\theta_{1}$ is also trace preserving. Induction now shows that $E_{M_{k}^{\prime} \cap M_{k+1}}\left(e_{k}\right)=\lambda 1, k \geq-1$, and thus $H\left(M_{k+1}, M_{k}\right)=$ $\ln \left[M_{k+1}: M_{k}\right]$.

To prove that $H\left(M_{n+k} \mid M_{k-1}\right)=\ln \left[M_{n+k}: M_{k-1}\right]$ it now suffices to prove that $H\left(M_{n} \mid N\right)=\ln \left[M_{n}: M\right]$ or, by $[3], E_{M_{n}^{\prime} \cap M_{2 n+1}}\left(f_{n}\right)=\lambda^{n+1} 1_{M_{2 n+1}}$. Since 
$M_{n}^{\prime} \cap M_{2 n+1} \subset M_{n-1}^{\prime} \cap M_{2 n+1} \subset \cdots \subset M^{\prime} \cap M_{2 n+1}$ we have $E_{M_{n}^{\prime} \cap M_{2 n+1}}=$ $E_{M_{n}^{\prime} \cap M_{2 n+1}} E_{M_{n-1}^{\prime} \cap M_{2 n+1}} \cdots E_{M^{\prime} \cap M_{2 n+1}}$. Since $e_{0}$ appears only once in $g_{n}^{0}$ and $E_{M^{\prime} \cap M_{2 n+1}}\left(e_{0}\right)=\lambda 1$ and $e_{i} \in M_{i-1}^{\prime}$, it follows that

$$
E_{M^{\prime} \cap M_{2 n+1}}\left(g_{n}^{0}\right)=\left(e_{n} \cdots e_{1} E_{M^{\prime} \cap M_{2 n+1}}\left(e_{0}\right)\right)\left(e_{n+1} \cdots e_{1}\right) \cdots\left(e_{2 n} e_{2 n-1} \cdots e_{n}\right) .
$$

Using now the same computations as in the proof of 2.6 it follows that

$$
E_{M^{\prime} \cap M_{2 n+1}}\left(g_{n}^{0}\right)=\lambda^{n+1} g_{n-1}^{1} .
$$

By induction it follows that

$$
\begin{aligned}
E_{M_{n}^{\prime} \cap M_{2 n+1}}\left(g_{n}^{0}\right) & =\lambda^{n+1} E_{M_{n}^{\prime} \cap M_{2 n+1}}\left(g_{n-1}^{1}\right)=\lambda^{n+1} E_{M_{n}^{\prime} \cap M_{2 n+1}} E_{M_{1}^{\prime} \cap M_{2 n+1}}\left(g_{n-1}^{1}\right) \\
& =\lambda^{n+1} \lambda^{n} E_{M_{n}^{\prime} \cap M_{2 n+1}}\left(g_{n-2}^{2}\right)=\cdots=\lambda^{(n+1)+n+\cdots 1} I
\end{aligned}
$$

and thus $E_{M_{n}^{\prime} \cap M_{2 n+1}}\left(f_{n}\right)=\lambda^{n+1} I$. Q.E.D.

3.2 CoROllary. Let $N \subset M$ be as in Theorem 3.1. Let $J_{n}$ be the canonical conjugation on $L^{2}\left(M_{n}, \tau\right)$. Suppose $M_{2 n+1}$ is represented on $L^{2}\left(M_{n}, \tau\right)$ so that to coincide with the basic construction of $N \subset M_{n}$. Then we have

(i) For every projection $f \in N^{\prime} \cap M_{n},\left[\left(M_{n}\right)_{f}: N_{f}\right]=\left[M_{n}: N\right] \tau(f)^{2}$.

(ii) The anti-isomorphism $N^{\prime} \cap M_{n} \ni x \mapsto J_{n} x J_{n} \in M_{n}^{\prime} \cap M_{2 n+1}$ is trace preserving.

(iii) For every $k \geq 0$ there exists a trace preserving isomorphism $N^{\prime} \cap M \ni x \mapsto$ $x^{\prime} \in M_{k-1}^{\prime} \cap M_{k}$ so that for every minimal projection $f \in N^{\prime} \cap M,\left[M_{f}: N_{f}\right]=$ $\left[\left(M_{k}\right)_{f}:\left(M_{k-1}\right)_{f^{\prime}}\right]$.

Proof. By 4.5 in [3] the condition $H\left(M_{n} \mid N\right)=\ln \left[M_{n}: N\right]$ is equivalent to the above conditions (i) and (ii). Then (iii) follows by (i), (ii) and by the fact that given any trace preserving anti-isomorphism between two finite-dimensional algebras there exists a trace preserving isomorphism between them which acts on the centers in the same way the anti-isomorphism does. Q.E.D.

\section{REFERENCES}

1. V. Jones, Index for subfactors, Invent. Math. 75 (1983), 1-25.

2. A. Ocneanu, in preparation.

3. M. Pimsner and S. Popa, Entropy and index for subfactors, Ann. Sci. Ecole Norm. Sup. 19 (1986), 57-106.

The National institute for Scientific and Technical Creation, DepartMENT OF MAThematics, BD. PACII 220, 79622 BuChaREST, ROMANia

Current address (M. Pimsner): Culmea Veche No 2, Sector 3, Bucharest, Romania

Current address (S. Popa): Department of Mathematics, University of California, Los Angeles, California 90024 